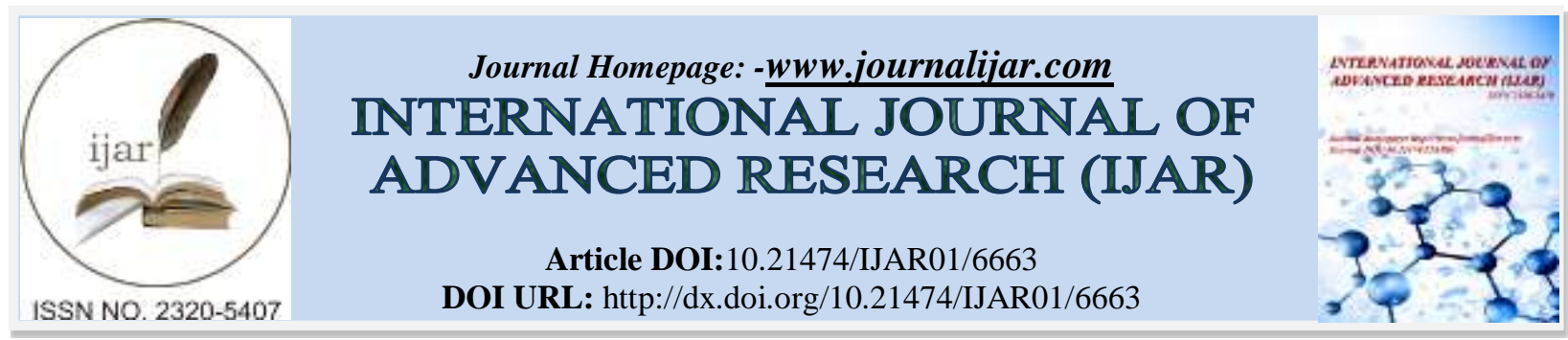

RESEARCH ARTICLE

\title{
INFLUENCING FACTORS OF ENVIRONMENTAL BEHAVIOR BASED ON THE THEORY OF PLANNED BEHAVIOR.
}

"Haibin Zhou' ${ }^{1}$ and Qiongyao $\mathrm{Li}^{2}$.

1. School of Public Affairs, University of Science and Technology of China No. 96, JinZhai Road, Baohe District, Hefei, Anhui, China.

2. School of Computer Science and Technology, Southwest University of Science and Technology, Mianyang, Si chuan, China.

\section{Manuscript Info}

Manuscript History

Received: 04 January 2018

Final Accepted: 06 February 2018

Published: March 2018

Keywords:-

Environmental Perception;

Environmental Behavior; Theory Of

Planned Behavior; Influencing Factors.

\section{Abstract}

This paper systematically reviews and summarizes individual environmental behavior, which is neglected in the existing research on environmental management, and concludes that such a behavior is influenced by three dimensions, namely, attitude, subjective norm, and behavior control according to the theory of planned behavior. On this basis, combining with literature research, self-efficacy is also taken as one of the influencing dimensions. The influencing factors of individual environmental behavior are then studied and discussed by questionnaire in light of foreign research results. The obtained data can provide suggestions and references for the government to protect the environment of backward minority areas in Western China.

Copy Right, IJAR, 2018,. All rights reserved.

\section{Introduction:-}

Catton WR and Dunlap RE (1978) reviewed previous studies and indicated that research on environmental management can be divided into two major categories. The first category is exploring how to formulate effective environmental management policies from the point of view of the government, which is on the macro level, and residents, which includes individual environmental perception and behavior. The second category is investigating how individuals participate in the government environmental management effectively, putting emphasis on the micro level. The effective combination of these two methods can make the research on environmental management efficient and effective.

As important participants in environmental management, individuals are the main bodies of environmental protection and environmental destruction, who not only cooperate but also conflict with the environmental management of the government. However, most of the existing environmental management researchers start from the macro level, such as policy research, ignoring individual behavior and influencing factors at the micro level. Therefore, this work conducts studies from the perspective of individuals, explores the factors that influence individual environmental behaviors, further understands the causes of various individual environmental behaviors, and attempts to provide references for governments to formulate all sorts of policies on environmental management. 


\section{Theoretical Basis and Scale Design:- Theory of Planned Behavior:-}

Ajzen and Fishbein (1988) built the theory of planned behavior on rational action theory. The theory of planned behavior states that intention is the key influencing factor of individuals' actual behavior. Behavior attitude, subjective norm, and perceived behavior control are the three variables that directly affect behavioral intention. On the basis of the theory of planned behavior, Cialdini (2001) and others studied the influence of belief on subjective norms and discovered that belief exerts an influence on all the three variables in the mentioned theory (Rivis and Sheeran, 2003). Therefore, belief is also included in the theoretical model of planned behavior, which is shown as follows:

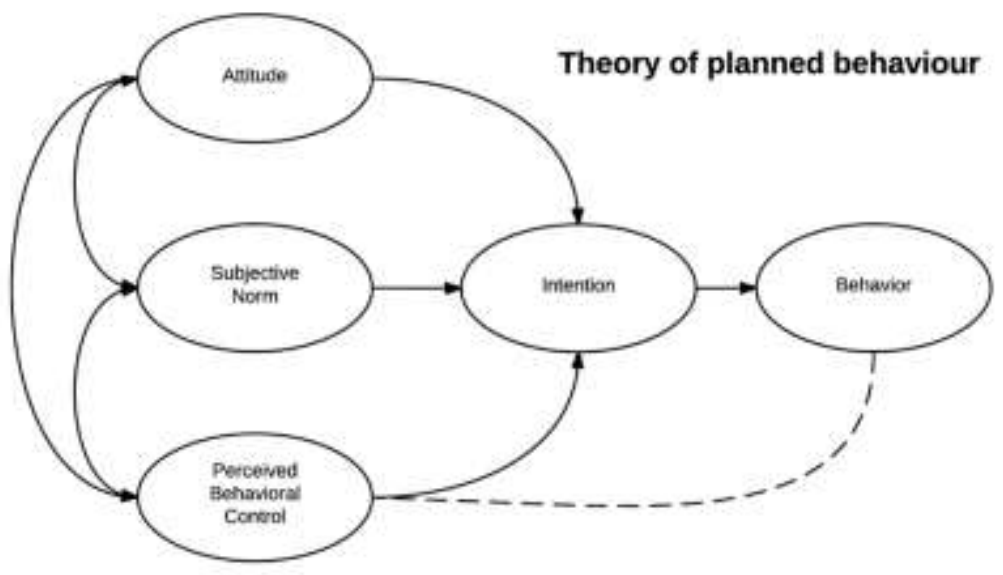

Figure 1:- Model of the Theory of Planned Behavior

In this model, an individual's behavior attitude is considered to be determined by behavior belief, which refers to the belief that a large number of different consequences may be produced by an individual's action (Ajzen, 1991). Normative beliefs contain two meanings. One is the individuals' understanding of important people's (family members, supervisors, colleagues, classmates, etc.) support or opposition to their certain acts, and the other one is the extent to which individuals follow these important people (Fishbein, 1975). Controlled belief refers to the perceptible factors that can promote or impede a specific environmental activity.

The theory of planned behavior is widely used in the study of environmental behavior, but restrictions exist on the scope of its application.

First, the objects of the study are the individuals who have rational behaviors. Behaviors (such as anger, excitement, and depression) influenced by the control of emotional factors, behaviors conforming to the opinion of the collective, and behaviors (such as parades and riots) following the crowd are excluded.

Second, the theory applies only to a specific individual behavior, not the behavior under broad conditions (Ajzen, 1977). For example, the theory of planned behavior is applicable for planting trees on the weekend afternoon by a person himself, which is not suitable for the broader scope, such as planting trees on the weekend.

Third, the consistency of an individual, that is, all the elements in the theoretical model, must describe the same individual.

The planned behavior is taken as an important theoretical basis for empirical research of this study. The individual environmental behavior model is modified accordingly, as shown in the Figure 2. 


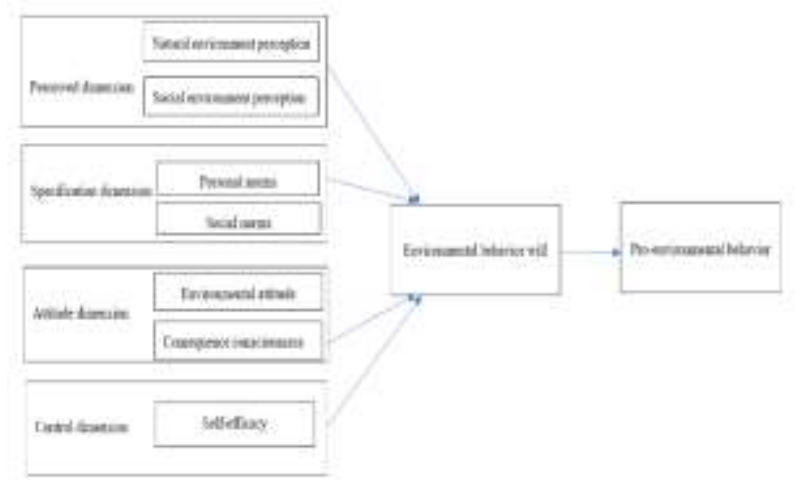

Figure 2:- Research Theory Model

\section{Scale Design:-}

The theory of planned behavior is taken as the theoretical basis to study the influencing factors of resident behavior. This study aims to obtain data for further measurement through questionnaire, which adopts Likert scale's rules to represent strongly disagree by the Arabic numeral 1 and strongly agree by 5 . The specific contents of the scale are as follows:

1. Measurement of residents' environmental behavior. Larson's measuring scale for environmental behavior (2015) is used as a reference. The scale consists of four dimensions, namely, economical lifestyle, land management, social environmentalism, and environmental citizenship behavior.

2. Measurement of residents' intention of environmental behavior. Drawing on the experience of Garling's scale of residents' intention of environmental behavior (2003), three items are included, namely, I will donate money to environmental organizations, I will sign a written petition about stricter environmental laws, and I will participate in demonstrations against companies that destroy the environment.

3. Measurement of residents' environmental perception. When conducting the study, scholars are identified to have conducted limited research on residents' environmental perception, and the corresponding measurement scales are also scarce. This study divides residents' environmental perception into two dimensions of natural and social environment perceptions, as adopted from Chinese scholar Xu Keshuai's (2008) scale.

4. Measurement of individual norms. Garling's (2003) measurement scale for individual norms, which contains four items, namely, environment protection is a moral obligation, I should protect the environment, protecting the environment is everyone's responsibility, and environmental problems cannot be ignored, are considered a reference.

5. Measurement of social norms. Three items, namely, most of the people who are important to me act in an environment-friendly way, most of the people who are important to me try to save resources, and most of my friends and companions are engaged in environment-friendly behavior, are taken from Bissing-Olson's (2016) measurement scale of social norms.

6. Measurement of consequence consciousness. The six items taken from Garling's (2003) measurement scale of consequence consciousness are the law to protect the environment restricts my choice and personal freedom, the environment protection will threaten the work of people like me, the impact of pollution on public health is more serious than we know, pollution in one country will jeopardize people all over the world, human behaviors are easy to break the balance of nature, and thousands of species are going to be extinct in the next few decades.

7. Measurement of environmental attitude. From Lee's (2014) measurement scale of environmental attitude, four items are included, namely, human beings abuse the environment, we will soon encounter a major ecological disaster if we continue to act like this, the balance of nature is fragile, and human beings should still abide by the laws of nature despite of growing power.

8. Measurement of self-efficacy. Under the enlightenment of Homburg's (2006) measurement scale of selfefficacy, four items, namely, I know how to take precautions against pollution in daily life, I can figure out how to deal with pollution in my daily life, I believe I can even deal with unexpected environmental problems, and I do not worry about the troubles caused by the global environmental problems because I am sure I can handle them, are mixed in the questionnaire. 


\section{Data Collection and Analysis:- \\ Data Collection:-}

This study selects the residents in the Heishui County of Aba State, Sichuan Province, which is a backward minority area of Western China, as research objects for the two following reasons.

1. The economic level of Heshui County, as a national impoverished county in China, is relatively backward. In 2014 , the per capita disposable income in this county was only $\$ 2,470$, and the per capita net income of farmers and herdsmen was $\$ 1,020$. The rapid development of local industry and tourism in recent years has led to a great contradiction between economic development and environmental protection in Heishui County, which fits the practical background of this study.

2. The geographical location and resident composition are unique. The landform of Heishui County is diverse and complex, with an average elevation of 3,544 $\mathrm{m}$, which results in its rich mineral resources and provides habitat for 161 species of wildlife. The agricultural population accounts for $90 \%$ of the inhabitants of Heshui County, and the Tibetan population is approximately $95 \%$. Therefore, the local residents' specific environmental behavior may be influenced by various factors, performing as a representative in the remote and backward areas of Western China.

In this study, a questionnaire survey is conducted in Heshui County for data collection. A total of 1,113 questionnaires are issued, and 656 of them are collected. The reason for the low response rate is that the local residents are less educated. Consequently, some of them cannot understand the questions, and some even cannot read.

The preprocessing of questionnaires led to 639 valid questionnaires, with an effective rate of $91.28 \%$. The demographic characteristics of the investigated samples are described in Table 1.

Table 1:- Demographic description of samples

\begin{tabular}{|c|c|c|c|c|c|}
\hline \multicolumn{2}{|c|}{ Demographic variables } & \multirow{2}{*}{\begin{tabular}{|l|}
$\begin{array}{l}\text { Composition } \\
\text { ratio }(\%)\end{array}$ \\
$51.1 \%$ \\
\end{tabular}} & \multicolumn{2}{|c|}{ Demographic variables } & \multirow{2}{*}{$\begin{array}{l}\text { Composition } \\
\text { ratio (\%) } \\
51.2 \%\end{array}$} \\
\hline \multirow{2}{*}{ Gender } & Male & & \multirow{5}{*}{$\begin{array}{l}\text { Average } \\
\text { monthly } \\
\text { household } \\
\text { income }\end{array}$} & $\leq 2000 \mathrm{RMB}$ & \\
\hline & Female & $48.9 \%$ & & 2001-4000 RMB & $19.1 \%$ \\
\hline \multirow{4}{*}{ Age } & $\leq 20$ years old & $2.1 \%$ & & 4001-5000 RMB & $17.5 \%$ \\
\hline & 21-30 years old & $33.3 \%$ & & 5001-6000 RMB & $10.3 \%$ \\
\hline & $31-40$ years old & $58.9 \%$ & & $\geq 6000 \mathrm{RMB}$ & $1.9 \%$ \\
\hline & $\geq 40$ years old & $5.7 \%$ & Position & Full-time student & $14.9 \%$ \\
\hline \multirow{3}{*}{$\begin{array}{l}\text { Length of } \\
\text { residence }\end{array}$} & $\leq 10$ years & $14.3 \%$ & & Business staff & $7.8 \%$ \\
\hline & $11-20$ years & $29.1 \%$ & & Farmer & $20.6 \%$ \\
\hline & $>20$ years & $57.6 \%$ & & Teacher and research worker & $17.7 \%$ \\
\hline \multirow{4}{*}{$\begin{array}{l}\text { Education } \\
\text { background }\end{array}$} & Primary school & $22 \%$ & & Private owner & $5.0 \%$ \\
\hline & Junior high school & $58.9 \%$ & & Personnel at institutions & $19.8 \%$ \\
\hline & High school & $15.6 \%$ & & Freelancer & $6.4 \%$ \\
\hline & $\begin{array}{ll}\begin{array}{l}\text { Specialty and } \\
\text { above }\end{array} & \text { and }\end{array}$ & $3.5 \%$ & & Others & $7.8 \%$ \\
\hline \multirow{2}{*}{$\begin{array}{l}\text { Type of } \\
\text { household } \\
\text { registration }\end{array}$} & $\begin{array}{l}\text { Agricultural } \\
\text { household }\end{array}$ & $60.9 \%$ & \multirow{2}{*}{$\begin{array}{l}\text { Current } \\
\text { residence }\end{array}$} & Country & $39.8 \%$ \\
\hline & $\begin{array}{l}\begin{array}{l}\text { Nonagricultural } \\
\text { household }\end{array} \\
\end{array}$ & $39.1 \%$ & & City (Town) & $60.2 \%$ \\
\hline
\end{tabular}

This study uses SPSS19.0 data statistical analysis software to conduct Cronbach's $\alpha$ test on related variables for testing reliability and validity. In the test of the pro-environmental behavior of residents, Cronbach's $\alpha$ value is 0.731, the Kaiser-Meyer-Olkin (KMO) value is 0.857 , and the factor loading matrix after each rotation is greater than 0.5. For the test on the environmental perception of residents, Cronbach's $\alpha$ value is 0.866 , the KMO value is 0.829 , and the corrected factor loading matrix of each item after rotation is greater than 0.5. Hence, reliability and validity are acceptable. 


\section{Results:-}

Demographic variables are analyzed using factor analysis of variance to determine natural environment perception, social environment perception, personal norm, social norm, personal consequence consciousness, social consequence consciousness, environmental attitude, self-efficacy, and environmental behavior. The results are as follows:

\section{Natural Environment Perception:-}

Significant differences exist in the natural environment perception among people with different genders, educational backgrounds, family average monthly incomes, and lengths of residence. Specifically, women are significantly lower in the natural environment perception than men; people living in the area for 10 years and below are significantly less aware of their natural environment than those who have lived in the area between 11 and 20 years; people with high school education, master's degree, and above obtain lower scores, whereas people with junior college and bachelor's degrees achieve higher scores.

Table 2:- Descriptive Statistics Table for Natural Environment Perception Based on Different Statistical Variables

\begin{tabular}{|c|c|c|c|c|c|}
\hline & Property & $\begin{array}{l}\text { Natural } \\
\text { environment } \\
\text { man value of } \\
\text { perception }\end{array}$ & $\begin{array}{l}\text { Standard } \\
\text { deviation }\end{array}$ & Sig. & Results \\
\hline Gender & $\begin{array}{l}\text { Female } \\
\text { Male }\end{array}$ & $\begin{array}{l}2.947 \\
3.157\end{array}$ & $\begin{array}{l}.785 \\
.719\end{array}$ & .100 & $\begin{array}{l}\text { Significant marginal differences } \\
\text { exist, and women are much } \\
\text { lower than men in natural } \\
\text { environment perception. }\end{array}$ \\
\hline Age & $\begin{array}{l}20 \text { years old and below } \\
\text { Between } 31 \text { and } 44 \text { years } \\
\text { old } \\
40 \text { years old and above }\end{array}$ & $\begin{array}{l}3.096 \\
3.017 \\
3.146 \\
2.750\end{array}$ & $\begin{array}{l}.787 \\
.755 \\
.693 \\
.825\end{array}$ & .856 & No significant difference \\
\hline $\begin{array}{l}\text { Length of } \\
\text { residence }\end{array}$ & $\begin{array}{l}10 \text { years and below } \\
\text { Between } 11 \text { and } 20 \text { years } \\
20 \text { years and above }\end{array}$ & $\begin{array}{l}2.974 \\
3.392 \\
3.034\end{array}$ & $\begin{array}{l}.795 \\
.792 \\
.618\end{array}$ & .085 & $\begin{array}{l}\text { Significant marginal differences } \\
\text { exist, and people living in the } \\
\text { area for } 10 \text { years and below are } \\
\text { significantly less aware of their } \\
\text { natural environment than those } \\
\text { who have lived in the area } \\
\text { between } 11 \text { and } 20 \text { years. }\end{array}$ \\
\hline $\begin{array}{l}\text { Educational } \\
\text { background }\end{array}$ & $\begin{array}{l}\text { Primary school } \\
\text { Junior middle school } \\
\text { Senior middle school } \\
\text { Junior college and above }\end{array}$ & $\begin{array}{l}3.000 \\
3.159 \\
3.135 \\
2.753\end{array}$ & $\begin{array}{l}.890 \\
.660 \\
.744 \\
.799\end{array}$ & .098 & $\begin{array}{l}\text { Significant marginal differences } \\
\text { exist, and people with high } \\
\text { school education, master's } \\
\text { degree, and above obtain lower } \\
\text { scores, whereas people with } \\
\text { junior college and bachelor's } \\
\text { degrees achieve higher scores. }\end{array}$ \\
\hline $\begin{array}{l}\text { Average } \\
\text { monthly } \\
\text { income of } \\
\text { family }\end{array}$ & $\begin{array}{l}2000 \text { yuan and below } \\
\text { Between } 2001 \text { and } 4000 \\
\text { yuan } \\
\text { Between } 4001 \text { and } 5000 \\
\text { yuan } \\
\text { Between } 5001 \text { and } 6000 \\
\text { yuan } \\
\text { Above } 6000 \text { yuan }\end{array}$ & $\begin{array}{l}2.944 \\
3.417 \\
2.814 \\
2.833 \\
2.988\end{array}$ & $\begin{array}{l}.811 \\
.673 \\
.868 \\
.692 \\
.725\end{array}$ & .029 & $\begin{array}{l}\text { The families with an average } \\
\text { monthly income of more than } \\
4,000 \text { yuan are significantly } \\
\text { lower than those with an average } \\
\text { monthly income between } 3,001 \\
\text { and } 4,000 \text { in the natural } \\
\text { environment perception. }\end{array}$ \\
\hline Position & $\begin{array}{l}\text { Full-time student } \\
\text { Worker } \\
\text { Peasant } \\
\text { Teacher and scientific } \\
\text { researcher } \\
\text { Private businessman }\end{array}$ & $\begin{array}{l}3.048 \\
2.908 \\
2.697 \\
3.197 \\
3.095 \\
3.242\end{array}$ & $\begin{array}{l}.881 \\
.668 \\
.670 \\
.521 \\
.560 \\
.814\end{array}$ & .283 & No significant difference \\
\hline
\end{tabular}




\begin{tabular}{|l|l|l|l|l|l|}
\hline & Institution staff & 2.704 & .701 & & \\
& Freelance & .858 & & \\
\hline Other & 3.152 & .833 & .435 & No significant difference \\
\hline $\begin{array}{l}\text { Type of } \\
\text { household } \\
\text { registration }\end{array}$ & Agricultural & 2.972 & .727 & & \\
\hline $\begin{array}{l}\text { Current } \\
\text { residence }\end{array}$ & Counagricultural & 3.082 & .869 & .171 & No significant difference \\
\hline
\end{tabular}

\section{Social Environment Perception:-}

Significant differences exist in social environment perception among people with different educational backgrounds, lengths of residence, and family average monthly incomes. Specifically, people living in the area for more than 20 years are significantly lower in social environment perception than those who have lived in the area for 10 years and below; people with junior middle school education are significantly lower in social environment perception than those with junior college education and above; population with an average monthly income between 5,001 and 6,000 yuan is significantly lower in social environment perception than the population who earn over 6,000 yuan; unlike the agricultural population, the nonagricultural-household-registered population is less aware of the social environment.

Table 3:- Descriptive Statistics Table for Social Environment Perception Based on Different Statistical Variables

\begin{tabular}{|c|c|c|c|c|c|}
\hline & Property & $\begin{array}{l}\text { Natural } \\
\text { Environment } \\
\text { Man Value of } \\
\text { Perception }\end{array}$ & $\begin{array}{l}\text { Standard } \\
\text { Deviation }\end{array}$ & Sig. & Results \\
\hline Gender & $\begin{array}{l}\text { Female } \\
\text { Male }\end{array}$ & $\begin{array}{l}3.066 \\
3.112 \\
\end{array}$ & $\begin{array}{l}.654 \\
.656 \\
\end{array}$ & 675 & No significant difference \\
\hline Age & $\begin{array}{l}21 \text { years old and below } \\
\text { Between } 31 \text { and } 44 \text { years } \\
\text { old } \\
40 \text { years old and above }\end{array}$ & $\begin{array}{l}3.162 \\
3.065 \\
2.875 \\
2.929 \\
\end{array}$ & $\begin{array}{l}.590 \\
.709 \\
.328 \\
1.111 \\
\end{array}$ & .633 & No significant difference \\
\hline $\begin{array}{l}\text { Length of } \\
\text { residence }\end{array}$ & $\begin{array}{l}11 \text { years and below } \\
\text { Between } 11 \text { and } 20 \text { years } \\
20 \text { years and above }\end{array}$ & $\begin{array}{l}3.209 \\
3.086 \\
2.835\end{array}$ & $\begin{array}{l}.640 \\
.594 \\
.652\end{array}$ & .012 & $\begin{array}{l}\text { People living for more than } 20 \\
\text { years have significantly lower } \\
\text { social environment perception } \\
\text { than those who have lived for } 10 \\
\text { years and below }\end{array}$ \\
\hline $\begin{array}{l}\text { Educational } \\
\text { background }\end{array}$ & $\begin{array}{l}\text { Primary school } \\
\text { Junior middle school } \\
\text { Senior middle school } \\
\text { Junior college and above }\end{array}$ & $\begin{array}{l}3.114 \\
2.851 \\
3.043 \\
3.373\end{array}$ & $\begin{array}{l}.548 \\
.488 \\
.688 \\
.599\end{array}$ & .025 & $\begin{array}{l}\text { People with junior middle school } \\
\text { education have significantly } \\
\text { lower social environment } \\
\text { perception than those with junior } \\
\text { college education and above }\end{array}$ \\
\hline $\begin{array}{l}\text { Average } \\
\text { monthly } \\
\text { income of } \\
\text { family }\end{array}$ & $\begin{array}{l}2000 \text { yuan and below } \\
\text { Between } 2001 \text { and } 4000 \\
\text { yuan } \\
\text { Between } 4001 \text { and } 5000 \\
\text { yuan } \\
\text { Between } 5001 \text { and } 6000 \\
\text { yuan } \\
\text { Above } 6000 \text { yuan }\end{array}$ & $\begin{array}{l}2.914 \\
3.013 \\
2.916 \\
2.540 \\
3.277\end{array}$ & $\begin{array}{l}.550 \\
.602 \\
.589 \\
.622 \\
.661\end{array}$ & .004 & $\begin{array}{l}\text { The population with an average } \\
\text { monthly income of } 5,001-6,000 \\
\text { yuan has much lower social } \\
\text { environment perception than } \\
\text { other groups. }\end{array}$ \\
\hline Position & \begin{tabular}{l}
\multicolumn{2}{l}{ Full-time student } \\
Enterprise grass-roots \\
Employees \\
Business management \\
personnel \\
Teacher and scientific \\
researcher
\end{tabular} & $\begin{array}{l}3.184 \\
3.227 \\
3.130 \\
3.117 \\
3.061 \\
2.918 \\
3.079 \\
\end{array}$ & $\begin{array}{l}.699 \\
.649 \\
.471 \\
.560 \\
.534 \\
.737 \\
.725 \\
\end{array}$ & .700 & No significant difference \\
\hline
\end{tabular}




\begin{tabular}{|c|c|c|c|c|c|}
\hline & $\begin{array}{l}\text { Private businessman } \\
\text { Institution staff } \\
\text { Freelance } \\
\text { Other }\end{array}$ & 3.143 & .507 & & \\
\hline $\begin{array}{l}\text { Type of } \\
\text { household } \\
\text { registration }\end{array}$ & $\begin{array}{l}\text { Agricultural } \\
\text { Non-agricultural }\end{array}$ & $\begin{array}{l}3.240 \\
3.026\end{array}$ & $\begin{array}{l}.576 \\
.675\end{array}$ & .076 & $\begin{array}{l}\text { The non-agricultural household } \\
\text { registered population is } \\
\text { significantly lower than the } \\
\text { agricultural population in social } \\
\text { environment perception. }\end{array}$ \\
\hline $\begin{array}{l}\text { Current } \\
\text { residence }\end{array}$ & $\begin{array}{l}\text { Countryside } \\
\text { City }\end{array}$ & $\begin{array}{l}3.060 \\
3.094\end{array}$ & $\begin{array}{l}.654 \\
.656\end{array}$ & .815 & No significant difference \\
\hline
\end{tabular}

\section{Consequence Consciousness:-}

Individual consequence perception between different genders, the types of household registration, and the places of residence have significant differences. Particularly, the individual consequence consciousness of women are significantly lower than that of men, non-agricultural household registered population is significantly lower than the agricultural household registered population, and the number of urban residents are significantly lower than that of rural residents.

Table 4:- Descriptive Statistics Table for Individual Consequence Consciousness Based on Different Statistical Variables

\begin{tabular}{|c|c|c|c|c|c|}
\hline & Property & \begin{tabular}{|l} 
Natural \\
Environment \\
Man Value of \\
Perception
\end{tabular} & $\begin{array}{l}\text { Standard } \\
\text { Deviation }\end{array}$ & Sig. & Results \\
\hline Gender & $\begin{array}{l}\text { Female } \\
\text { Male }\end{array}$ & $\begin{array}{l}2.396 \\
2.681\end{array}$ & $\begin{array}{l}.978 \\
1.014\end{array}$ & .091 & $\begin{array}{l}\text { Women have significantly lower } \\
\text { individual consequence } \\
\text { consciousness than men. }\end{array}$ \\
\hline Age & $\begin{array}{l}22 \text { years old and below } \\
\text { Between } 31 \text { and } 44 \text { years } \\
\text { old } \\
40 \text { years old and above }\end{array}$ & $\begin{array}{l}2.587 \\
2.538 \\
2.188 \\
2.500 \\
\end{array}$ & $\begin{array}{l}1.079 \\
.983 \\
.790 \\
.707\end{array}$ & .781 & No significant difference \\
\hline $\begin{array}{l}\text { Length of } \\
\text { residence }\end{array}$ & $\begin{array}{l}12 \text { years and below } \\
\text { Between } 11 \text { and } 20 \text { years } \\
20 \text { years and above }\end{array}$ & $\begin{array}{l}2.512 \\
2.700 \\
2.500\end{array}$ & $\begin{array}{l}1.000 \\
1.140 \\
.953\end{array}$ & .732 & No significant difference \\
\hline $\begin{array}{l}\text { Educational } \\
\text { background }\end{array}$ & $\begin{array}{l}\text { Primary school } \\
\text { Junior middle school } \\
\text { Senior middle school } \\
\text { Junior college and above }\end{array}$ & $\begin{array}{l}2.500 \\
2.364 \\
2.548 \\
2.629 \\
\end{array}$ & $\begin{array}{l}.866 \\
.915 \\
1.002 \\
1.110\end{array}$ & .820 & No significant difference \\
\hline $\begin{array}{l}\text { Average } \\
\text { monthly } \\
\text { income of } \\
\text { family }\end{array}$ & $\begin{array}{l}2000 \text { yuan and below } \\
\text { Between } 2001 \text { and } 4000 \\
\text { yuan } \\
\text { Between } 4001 \text { and } 5000 \\
\text { yuan } \\
\text { Between } 5001 \text { and } 6000 \\
\text { yuan } \\
\text { Above } 6000 \text { yuan }\end{array}$ & $\begin{array}{l}2.633 \\
2.797 \\
2.735 \\
2.333 \\
2.368\end{array}$ & $\begin{array}{l}1.043 \\
1.128 \\
1.002 \\
1.000 \\
.921\end{array}$ & .263 & No significant difference \\
\hline Position & $\begin{array}{l}\text { Full-time student } \\
\text { Worker } \\
\text { Peasant } \\
\text { Teacher and scientific } \\
\text { researcher } \\
\text { Private businessman } \\
\text { Institution staff } \\
\text { Freelance } \\
\end{array}$ & \begin{tabular}{|l|}
2.714 \\
2.241 \\
2.318 \\
2.409 \\
2.500 \\
2.702 \\
2.333 \\
2.864 \\
\end{tabular} & $\begin{array}{l}.930 \\
.872 \\
.681 \\
.831 \\
.707 \\
1.220 \\
1.199 \\
.924 \\
\end{array}$ & .482 & No significant difference \\
\hline
\end{tabular}




\begin{tabular}{|c|c|c|c|c|c|}
\hline & Other & & & & \\
\hline $\begin{array}{l}\text { Type of } \\
\text { household } \\
\text { registration }\end{array}$ & $\begin{array}{l}\text { Agricultural } \\
\text { Non-agricultural }\end{array}$ & $\begin{array}{l}2.793 \\
2.430\end{array}$ & $\begin{array}{l}1.107 \\
.94286\end{array}$ & .051 & $\begin{array}{l}\text { The individual consequence } \\
\text { consciousness of non- } \\
\text { agricultural household registered } \\
\text { population is significantly lower } \\
\text { than that of agricultural } \\
\text { household registered population. }\end{array}$ \\
\hline $\begin{array}{l}\text { Current } \\
\text { residence }\end{array}$ & $\begin{array}{l}\text { Countryside } \\
\text { City }\end{array}$ & $\begin{array}{l}3.125 \\
2.415\end{array}$ & $\begin{array}{l}1.066 \\
.950\end{array}$ & .001 & $\begin{array}{l}\text { The individual consequence } \\
\text { consciousness of urban residents } \\
\text { is significantly lower than that of } \\
\text { rural residents. }\end{array}$ \\
\hline
\end{tabular}

Self-efficacy:-

Self-efficacy has significant differences among people of different ages. Self-efficacy gradually decreases with the growth of age.

Table 5:- Descriptive Statistics Table for Self-efficacy Based on Different Statistical Variables

\begin{tabular}{|c|c|c|c|c|c|}
\hline & Property & $\begin{array}{l}\text { Natural } \\
\text { Environment } \\
\text { Man Value of } \\
\text { Perception }\end{array}$ & $\begin{array}{l}\text { Standard } \\
\text { Deviation }\end{array}$ & Sig. & Results \\
\hline Gender & $\begin{array}{l}\text { Female } \\
\text { Male }\end{array}$ & $\begin{array}{l}3.278 \\
3.266\end{array}$ & $\begin{array}{l}.669 \\
.839\end{array}$ & .925 & No significant difference \\
\hline Age & $\begin{array}{l}23 \text { years old and below } \\
\text { Between } 31 \text { and } 44 \text { years } \\
\text { old } \\
40 \text { years old and above }\end{array}$ & $\begin{array}{l}3.494 \\
3.169 \\
2.958 \\
2.833\end{array}$ & $\begin{array}{l}.734 \\
.768 \\
.486 \\
.236\end{array}$ & .044 & $\begin{array}{l}\text { Self-efficacy gradually decreases } \\
\text { with the growth of age. }\end{array}$ \\
\hline $\begin{array}{l}\text { Length of } \\
\text { residence }\end{array}$ & $\begin{array}{l}13 \text { years and below } \\
\text { Between } 11 \text { and } 20 \text { years } \\
20 \text { years and above }\end{array}$ & $\begin{array}{l}3.362 \\
3.050 \\
3.197 \\
\end{array}$ & $\begin{array}{l}.791 \\
.880 \\
.571\end{array}$ & .195 & No significant difference \\
\hline $\begin{array}{l}\text { Educational } \\
\text { background }\end{array}$ & $\begin{array}{l}\text { Primary school } \\
\text { Junior middle school } \\
\text { Senior middle school } \\
\text { Junior college and above }\end{array}$ & $\begin{array}{l}2.667 \\
3.167 \\
3.285 \\
3.409\end{array}$ & $\begin{array}{l}1.054 \\
.726 \\
.731 \\
.768\end{array}$ & .196 & No significant difference \\
\hline $\begin{array}{l}\text { Average } \\
\text { monthly } \\
\text { income of } \\
\text { family }\end{array}$ & $\begin{array}{l}2000 \text { yuan and below } \\
\text { Between } 2001 \text { and } 4000 \\
\text { yuan } \\
\text { Between } 4001 \text { and } 5000 \\
\text { yuan } \\
\text { Between } 5001 \text { and } 6000 \\
\text { yuan } \\
\text { Above } 6000 \text { yuan }\end{array}$ & $\begin{array}{l}3.133 \\
3.354 \\
3.294 \\
3.185 \\
3.270\end{array}$ & $\begin{array}{l}.664 \\
.743 \\
.841 \\
.648 \\
.784\end{array}$ & .909 & No significant difference \\
\hline Position & $\begin{array}{l}\text { Full-time student } \\
\text { Worker } \\
\text { Peasant } \\
\text { Teacher and scientific } \\
\text { researcher } \\
\text { Private businessman } \\
\text { Institution staff } \\
\text { Freelance } \\
\text { Other }\end{array}$ & $\begin{array}{l}3.429 \\
3.115 \\
3.000 \\
3.515 \\
3.286 \\
3.278 \\
2.926 \\
3.667\end{array}$ & $\begin{array}{l}.700 \\
.747 \\
.760 \\
.861 \\
.300 \\
.750 \\
.954 \\
.683\end{array}$ & .208 & No significant difference \\
\hline $\begin{array}{l}\text { Type of } \\
\text { household } \\
\text { registration }\end{array}$ & $\begin{array}{l}\text { Agricultural } \\
\text { Non-agricultural }\end{array}$ & $\begin{array}{l}3.488 \\
3.183\end{array}$ & $\begin{array}{l}.885 \\
.679\end{array}$ & .029 & \\
\hline Current & Countryside & 3.500 & .702 & .104 & No significant difference \\
\hline
\end{tabular}


residence

City

3.225

.759

Other Aspects:-

Individual norm: All demographic variables have no significant differences in individual norm.

Social norm: All demographic variables have no significant differences in social norm.

Social consequence consciousness: All demographic variables have no significant differences in social consequence consciousness.

Environmental attitude: All demographic variables have no significant differences in environmental attitude.

Environmental behavior intention: All demographic variables have no significant differences in environmental behavior intention.

Environmental behavior: All demographic variables have no significant differences in environmental behavior.

\section{Discussion:-}

Discussion on the Differences of the Environmental Perception of Residents:-

First, gender causes differences in the environmental perception of residents. The results in this study show that the influence of gender on environmental perception is mainly embodied in the natural environment perception, whereas the difference in social environment perception is not evident. Arcury (1990) indicated that men are superior to women in environmental perception, which is in line with the results in this study. However, some studies emphasize that environmental perception caused by genders have no significant statistical difference, that is, gender does not influence the results of environmental perception (Hunter, 2004).

In the literature research, some scholars used highly generalized questions, which do not discriminate between natural and social environment, thus, arousing misunderstanding among respondents is easy. That is, changes in the environment should be seen separately, and the natural and social environment should be measured individually. Measuring individuals by using general questions may cause misinterpretation and result in great differences compared with the conclusions of different scholars.

Second, the length of residency causes differences in the environmental perception of residents. The results show that the length of residency has a great influence on the environmental perception of local residents. Different natural environment perceptions of people who have been living in Heishui County for several years may be caused by local consciousness. Agnew (1987) defined sites with three elements, namely, fixed coordinates or locations, social environment, and local consciousness. Among these elements, local consciousness refers to the emotional link that connects individuals to a certain place. Stedman (2002) emphasized that the environmental perception and behavior of residents are closely related to the length of their residency. They are more sensitive to environmental perception when they live longer. Chinese scholars, Wang Jianming (2015), clarified that people who have lived long in one place may feel the changes when they occur.

Third, difference in educational background causes variations in the environmental perception of residents. The analysis shows that people with various educational backgrounds have significant differences in natural and social environment perception. Less educated people are more sensitive to social environment perception, whereas more educated people are more sensitive to natural environment perception. Well-educated people are likely to have more knowledge regarding environment protection than people who know nothing about this issue. Thus, well-educated people intend to care more about environmental protection. However, the results in Grendstad and Wollebaek (1998) show that people with low education level are more concern about the environment. Therefore, the academic circle has not formed a unified opinion on the impact of educational background on environmental perception.

As for the actual situation in the local area, people with higher education usually work in institutions or companies or move to the urban district, indicating that they do not engage in productive labor in the natural environment directly. Compared with the impressions of natural environment years ago, they believe great changes have taken place in the natural environment. On the contrary, less educated people often work in rural areas or grass-root units. 
Accordingly, they are easily impressed by the changes of social environment, such as neighborhood relationships and public facilities.

Fourth, income causes differences in the environmental perception of residents. The average income of urban residents is normally higher than that of the rural residents. Urban residents have personally experienced the changes of the urban social environment so they may think that the change in urban construction is minimal. Similarly, residents who live in the countryside may think that the changes of the natural environment are relatively small. In this study, people who earn more than 4,000 yuan a month are less aware of the natural environment than those with lower income. The data show that people with an average monthly income of 4,001-5,000 yuan have significantly lower social environment perception than other groups. People who earn more than 5,000 yuan should also show significant correlation based on the general situation. The possible reason is that local families who earn between 4,001 and 5,000 can be labeled as high-income families, hence, less significance is shown among population with a monthly income of more than 5,000 yuan probably because the members of this group is relatively few.

\section{Discussion on the Difference of Consequence Consciousness:-}

On the one hand, gender causes difference in consequence consciousness. Kennemer (2002), Nakamura (2006), and other researchers have suggested that men are more aware of social development than women, and men are more likely to think about problems from a rational point of view than women. In the specific case of Heishui County, the social status of women is far lower than that of men, and almost all the housework and farm work are undertaken by women. Under harsh nature and impoverished economy, women hardly have time, energy, and cognitive ability to perceive the environment, resulting in differences in consequence consciousness.

On the other hand, the type of household and residence registration causes difference in consequence consciousness. In terms of differences in the type of household and residence registration, the results show that population with non-agricultural registration and urban residents have low consequence consciousness. A large number of local rural residents and herdsmen have moved to the urban areas from remote plateaus and mountain areas in recent years. The current living conditions of the relocated population have been greatly improved compared with their previous living conditions and their focus has shifted to increasing their income. Therefore, local residents have kept reducing their awareness on the consequences of environmental behaviors, which have been repeatedly banned by the local government, to pursue economic benefits. This phenomenon explains the non-agricultural registration of the population and the decreasing consequence consciousness of urban residents.

\section{Difference of Self-efficacy:-}

Data show that the self-efficacy of local residents is decreasing with the growth of age. First, the majority of the local residents can accept complete, compulsory education for nine years and improve their cognition of the environment with the improvement of the economic situation. Some of the senior residents cannot even read the Chinese characters at all and do not receive formal education, hence, their self-efficacy is quite low. Second, young local residents would often want to change the situation of poverty by their own efforts and are not willing to continue to live a life as poor as their ancestors. Senior residents tend to have no clear goals and plans for their future due to their lack of certain proficiency and corresponding available resources. As a result, the data indicate that the correlation between the self-efficacy and age of residents is reasonable.

\section{Research Limitations and Future Prospects:-}

Local residents in Heishui County, as the research objects, are tested for their environment perception and the impact of various factors on environmental behavior intention and environmental behavior. However, during the actual investigation and analysis, the survey sample is found to be too concentrated on the less educated population and focuses less on local people with relatively high academic qualifications and those who have left for further study. Thus, the number of samples is still insufficient. The level of educational level influences environmental perception and behavior. Accordingly, the environmental management policy of the government also needs to make corresponding adjustments. In this respect, the limitations of this study are obvious. Future research can select these groups as objects to increase the applicability of their results. In addition, under the constraints of looking for proper objects, the sample volume collected in this study is not quite sufficient and may not represent all classes. That is, other pieces of evidence are needed to promote this conclusion further. The number of samples should be increase in the future research to improve the accuracy of the model and the application scope of the research conclusions. At the same time, the current research is inadequate compared with the complex research on the relationship of variables. Moreover, the investigation of related factors should be increased in future research. 
This study adopts the form of questionnaire survey, which can be influenced by subjective factors, such as emotions. The results obtained may not be $100 \%$ true due to the uncontrollable situation when answering the questions. In future research, more tools should be added. The theoretical model and assumptions are examined by statistical inference of cross-sectional data set because of the limitations of the level and conditions of research. Consequently, proving that the research variables have strict causality is difficult. Follow-up studies can further test relevant conclusions through longitudinal research.

\section{References:-}

1. Ajzen, I. 1991. The theory of planned behavior. Organizational behavior and human decision processes, 50,179211.

2. Ajzen, I. and Driver BL. 1992. Contingent value measurement: on the nature and meaning of willingness to pay. Journal of Consumer Psychology,1, 297-316.

3. Bamberg S, and Schmidt P. 2003. Incentives, morality, or habit? Predicting students' car use for university routes with the models of Ajzen, Schwartz, and Triandis. Environmental and behavior, 35, 264-285.

4. Bissing-olson M J., and Fielding K S., and Iyer A. 2016. Experiences of pride, not guilt, predict proenvironmental behavior when pro-environmental descriptive norms are more positive. Journal of Environmental Psychology, 45, 145-153.

5. Guo, Lingxia, and Feng, Jianmin, and Sun, BO. 2015. Environmental Perception of Farmers and Its Influence Factors in Ecological Fragile Area of Northern Shaanxi Province. Bulletin of Soil and Water Conservation, 35, 235-241.

6. Lee Y, and Kim S, and Kim M. 2014. Antecedents and interrelationships of three types of pro-environmental behavior. Journal of Business Research, 67, 2097-2105.

7. Onwezen M C, and Antonides G, and Bartels J. 2013. The norm Activation Model: An exploration of the functions of anticipated pride and guilt in pro-environmental behavior. Journal of Economic Psychology, 39,141-153.

8. Stern P. C. 2000. New environmental theories: toward a coherent theory of environmentally significant behavior. Journal of Social Issues, 56, 407-424.

9. Wynveen CJ. and Sutton S G. 2015. Engaging the public in climate change-related pro-environmental behaviors to protect coral reefs: The role of public trust in management agency. Marine policy, 53, 131-140.

10. Xu, Lizhong, and Chen Fang, and Yang Jing, and Zhong, Manxiu, and Hu Jun. 2013. Motivation Behind Contingent Valuation in the Context of the Planned Behavior Theory. Journal of Fujian Normal University (Natural Science Edition), 5,87-93.

11. Yu, Wei. 2010. Study on the Formation Mechanism of Urban Environmental Behavior From TPB:Investigation from Large and Medium-sized Cities in Shandong Province.Ecological Economy, 6,160-163. 